\title{
Design, fabrication, and characterisation of wire grid polarizers for the deep UV spectral range
}

Rodríguez-de Marcos, Luis, Ong Bin, Leong, Citra Asmara, Teguh, Heussler, Sascha Pierre, Guerrero , Albert, et al.

Luis Rodríguez-de Marcos, Leong Ong Bin, Teguh Citra Asmara, Sascha Pierre Heussler, Albert Guerrero, Roser Mas , Xavier Borrise, Mark B. H. Breese, Andrivo Rusydi, "Design, fabrication, and characterisation of wire grid polarizers for the deep UV spectral range," Proc. SPIE 10691, Advances in Optical Thin Films VI, 1069124 (5 June 2018); doi: 10.1117/12.2314459 


\title{
Design, fabrication and characterization of wire grid polarizers for the deep UV spectral range
}

\author{
Luis Rodríguez-de Marcos ${ }^{1}$, Ong Bin Leong ${ }^{1,2}$, Teguh Citra Asmara ${ }^{1}$, Sascha Pierre Heussler ${ }^{1}$, \\ Albert Guerrero ${ }^{3}$, Roser Mas $^{3}$, Xavier Borrise ${ }^{4}$, Mark B. H. Breese ${ }^{1,2}$, and Andrivo Rusydi ${ }^{1,2}$ \\ ${ }^{1}$ Singapore Synchrotron Light Source, National University of Singapore, 5 Research Link, \\ 117603 Singapore \\ ${ }^{2}$ Physics Department, National University of Singapore, 2 Science Drive 3, 117542 Singapore \\ ${ }^{3}$ Institute of Microelectronics of Barcelona (CSIC), U. Autonoma de Barcelona, 08193 \\ Bellaterra, Spain \\ ${ }^{4}$ Catalan Institute of Nanoscience and Nanotechnology (CSIC) and The Barcelona Institute of \\ Science and Technology, Campus UAB, 08193 Bellaterra, Spain
}

\begin{abstract}
In this communication, we show preliminary results on transmissive $\mathrm{TiO}_{2}$ wire-grid polarizers (WGP) operating in the deep ultraviolet (DUV) range. WGP are devices based on strips of materials with large values of the modulus of the dielectric constant along with high absorption in the operational range. The merit function $\Pi$ is introduced as a new tool to find the optimum material for WGPs in a given spectral range. The experimental dielectric constant of $\mathrm{TiO}_{2}$ thin films deposited by pulsed laser deposition are obtained through spectroscopic ellipsometry, and the $\Pi$ function indicates that $\mathrm{TiO}_{2}$ is the best candidate for WGP in the DUV range when it is compared with other oxides. Once the material selection for WGP is done, we present and compare two different design approaches for WGP: one using an effective medium theory for the periodic structure, and the second using finite-difference time-domain (FDTD) analysis. A prototype of WGP is fabricated by electron beam (e-beam) lithography followed by lift-off process; the topography of the sample is analyzed by AFM, and we found noticeable deviations in the grating from the designed values. In preliminary characterization work the effective dielectric constant in two perpendicular orientations is obtained by ellipsometry and the contrast is compared with the design.
\end{abstract}

\section{INTRODUCTION}

Manipulation of polarization of electromagnetic radiation in the DUV spectral range is a key requirement for optical techniques such as spectrophotometry, ellipsometry, and polarimetry. Wire grid polarizers are zero-order sub-wavelength gratings with parallel wires, which transmit (absorb) radiation with the electric field vector perpendicular, or TM, (parallel, or TE) to wires. WGPs present many advantages in comparison with polarizers based on Brewster's angle or on crystal birefringence, such as normal-incidence operation (with no beam deviation), large acceptance angles, compact thickness, and short propagation distances. Nowadays WGP are a well-established technology in the visible and IR ranges, where wire geometries are usually in the micron scale. With advancements in nanofabrication, the 
generation of wires in the nanometre scale becomes achievable, pushing the feasibility of these devices towards the DUV range.

Efficient WGP requires materials with large values of $|\varepsilon|$ along with high absorption; this requirement will be further discussed in section 3 . In the visible and infrared ranges, WGPs are commonly based on metals, with high absorption due to their Drude-like behaviour. However, WGPs based on metals are less efficient in the DUV, as the metal plasma frequency is either longwards of DUV or, for those metals whose plasma frequency is at shorter wavelengths, they present low absorption values in DUV range. Contrarily, wide bandgap high-index oxides, such as $\mathrm{TiO}_{2}, \mathrm{SrTiO}_{3}$ or $\mathrm{Nb}_{2} \mathrm{O}_{5}$, are promising candidates for WGP in DUV, due to their strongly absorbent behaviour shortwards of their cut-off wavelength. Oxides present additional advantages in comparison with metals: They do not need to be protected against oxidation or corrosion, they have good mechanical properties, small surface roughness, and its transparency in the visible and infrared range makes them suitable for applications where heat must be damped. Moreover, the tuneability of the absorption spectra that has been recently discovered ${ }^{1}$ in $\mathrm{TiO}_{2}$ thin films due to doping with Ta opens a new horizon for WGPs, whose performance is nowadays mainly limited by the optical properties of available materials.

WGPs have been widely studied in the visible and IR spectral ranges, and there is a relatively large number of research articles reporting design, fabrication and characterization of these devices. On the other hand, there is few data in the literature reporting WGPs for DUV range: Asano et al. $^{2}$ developed WGPs based on $\mathrm{Cr}_{2} \mathrm{O}_{3}$ fabricated through double patterning lithography process, Gardner et al. ${ }^{3}$ patented WGPs based on $\mathrm{TiO}_{2}$ and on $\mathrm{Nb}_{2} \mathrm{O}_{5}$, however nothing is said in his patent about the fabrication process, and Siefke et al. ${ }^{4}$ reported WGPs based on $\mathrm{TiO}_{2}$ fabricated through self-aligned double patterning lithography process.

In this proceeding, we present designs and preliminary results on $\mathrm{TiO}_{2}$ WGP optimized for the DUV range. Section 2 describes sample preparation and characterization techniques. Section 3 introduces the merit function $\Pi, \mathrm{TiO}_{2}$ optical constants are shown, and we detail the WGP design procedure following two different paths. Section 4 details the fabrication of WGP through e-beam lithography followed with lift-off process. Finally, in section 5 preliminary results on optical performance of the device are shown.

\section{EXPERIMENTAL TECHNIQUES}

$\mathrm{TiO}_{2}$ thin films were prepared in a state-of-the-art pulsed laser deposition system (NEOCERA); this system is component of the SUV beamline in SSLS facility. $\mathrm{TiO}_{2}$ targets with a purity of $99.999 \%$ from LATECH were ablated using a Continuum-Surelite III solid state laser operating with the fourth harmonic at $266 \mathrm{~nm}$, with pulse frequencies of $10 \mathrm{~Hz}$ and laser power of $100 \mathrm{~mJ} / \mathrm{cm}^{2}$. The deposition chamber was pumped by turbo molecular and diaphragm pumps; in order to accelerate degassing, deposition chamber was baked out up to $\sim 130{ }^{\circ} \mathrm{C}$. Base pressure was $6.5 \cdot 10^{-9}$ Torr. The angle of incidence of the laser on target was $45^{\circ}$, and the target-to-substrate distance was $\sim 100 \mathrm{~mm}$. Target rastering and rotation was used in order to 
uniformly ablate all the target surface. Thin films were deposited either on $10 \mathrm{~mm} \times 10 \mathrm{~mm} \times 1$ $\mathrm{mm}$ double-side polished fused silica JGS-1 substrates (for WGP) and on one-side polished zcut quartz substrates (for optical constants determination), both from LATECH and both polished to a surface roughness of less than $5 \AA$. Substrates rotated during reposition at $\sim 29$ $\mathrm{deg} / \mathrm{s}$. An Inconel alloy absorber was placed in contact with the backside of substrate, and it was heated with a $140 \mathrm{~W}$ laser heater. Films on quartz were deposited on substrates heated at $500{ }^{\circ} \mathrm{C}$, whereas $\mathrm{TiO}_{2}$ for WGP was deposited at room temperature, but annealed at $500{ }^{\circ} \mathrm{C}$ after the lift-off process. Heating and cooling rates were set to $15^{\circ} \mathrm{C} / \mathrm{min}$ either for deposition or for annealing. Substrate temperature was controlled and monitored by an optical pyrometer. Deposition was made in $\mathrm{O}_{2}$ partial pressure of $10 \mathrm{mTorr}$, controlled by a mass flow controller.

The complex dielectric constant and thickness of $\mathrm{TiO}_{2}$ thin films deposited on quartz substrates were determined with the SENTECH SE850 Spectroscopic Ellipsometer, available at SSLS. In order to determine $\mathrm{TiO}_{2}$ dielectric constant, samples were measured at $70^{\circ}$ from normal incidence in the 200-950 nm spectral range. The effective TE and TM dielectric constants of the patterned WGP were measured at $50^{\circ}$ from normal incidence in the same spectral range. For the latter, $\psi$ and $\Delta$ was obtained in two perpendicular orientations, one parallel and the other perpendicular to the $\mathrm{TiO}_{2}$ lines. Focusing lenses were installed at the exit of the polarizer arm and at the entrance of the analyser arm, in order to reduce the spot size to less than $1 \mathrm{~mm} x$ $1 \mathrm{~mm}$ at sample surface when operating at $50^{\circ}$.

Topography measurements were performed with a Bruker Dimension Icon AFM with ScanAsyst, using tapping mode with a VESPA-v2 tip. This instrument is available at Physics department, NUS.

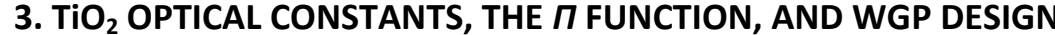

\section{$\underline{3.1 \text { Optical properties of } \mathrm{TiO}_{2}}$}

Optical constants are fundamental parameters for the design of WGP. The complex dielectric constant of $\mathrm{TiO}_{2}$ films was obtained through spectroscopic ellipsometry. An uncoated one-side polished quartz substrate was previously characterized and compared against reported $\mathrm{SiO}_{2}$ optical constants by Philipp ${ }^{5}$. Figure 1 (left) displays the ellipsometry parameters $\tan (\psi) e^{i \Delta}=r_{p} / r_{s}$ measured at $70^{\circ}$ from normal incidence of a 105-nm thick $\mathrm{TiO}_{2}$ sample deposited on quartz, and the fit with a Tauc-Lorentz model ${ }^{6}$. Figure 1 (right) displays the dielectric constants of $\mathrm{TiO}_{2}$ thin film derived from the model. 

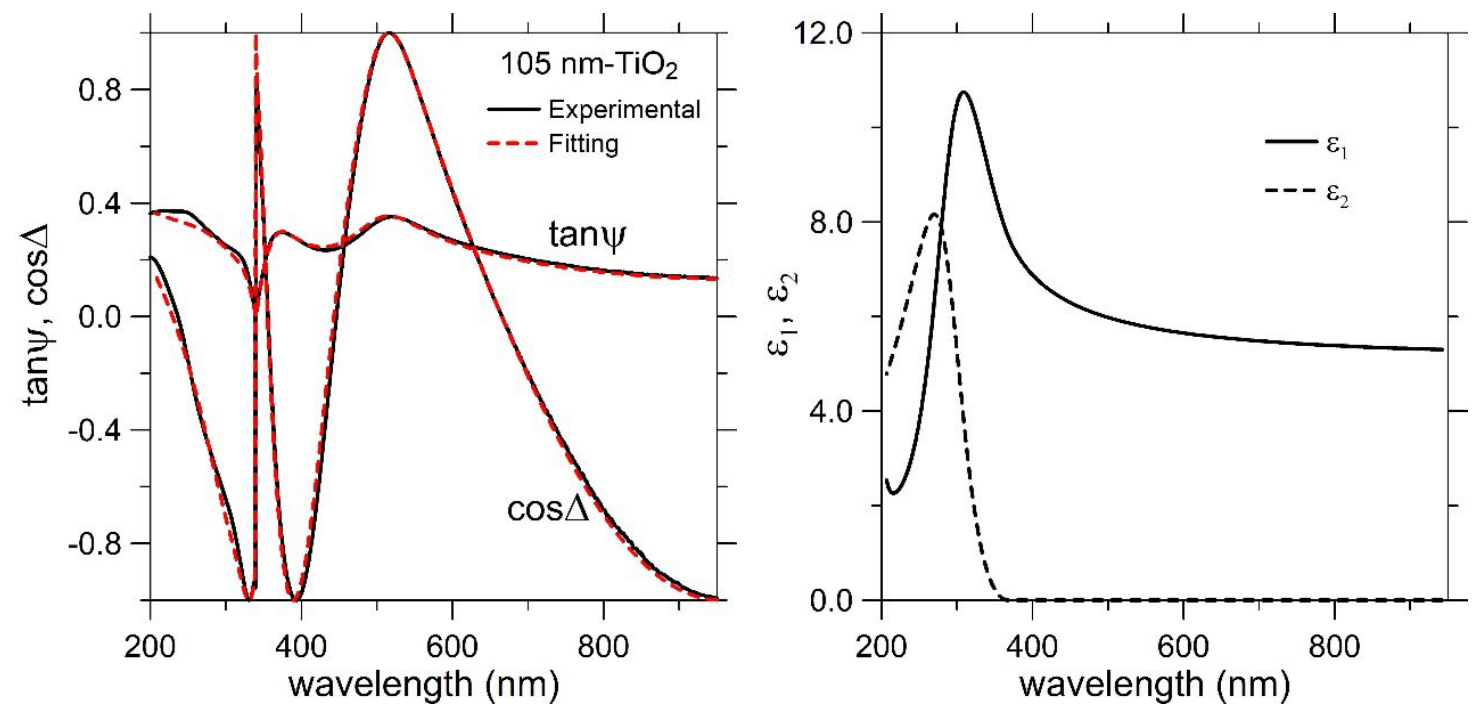

Fig. 1. (Left) Experimental values of $\tan (\psi)$ and $\cos (\Delta)$ at $70^{\circ}$ incidence angle, and the fitting with a TaucLorentz model. (Right) Complex dielectric function of $\mathrm{TiO}_{2}$ extracted from model

\subsection{Material selection with the $\Pi$ function}

The efficiency of WGPs is strongly dependent on effective dielectric constant contrast "seen" by TE and TM waves. The optical response of the grating to TE and TM polarizations is opposite: for TM wave, the grating acts as a relatively transparent anti-reflection (AR) coating, whereas for TE wave the grating acts like an absorbent medium. As will be demonstrated in subsection 3.3 with the EMT, if the grating material has small values of $|\varepsilon|$ in the range of interest, the effective dielectric constant contrast will be poor regardless of the choice of grating parameters. The same reasoning can be applied to absorption.

We propose the function $\Pi=|\varepsilon| \cdot \operatorname{Im}(\varepsilon)$ as a new tool to evaluate the viability of materials for WGP in a desired spectral range. This function combines in a simple way the two main requirements for WGP materials. We also propose the threshold $\Pi \geq \frac{\lambda}{5 \mathrm{~nm}}$ as the condition that any material must satisfy to obtain efficient WGPs in the target spectral range. This criterion somehow takes into account the current state of the art of transmissive polarizers in different spectral ranges, i.e. an extinction ratio of 20 is very good at $120 \mathrm{~nm}$ but the same ratio would be mediocre in the visible. Figure 2 depicts $\Pi$ functions calculated for several oxides in the DUV and adjacent ranges. $\mathrm{TiO}_{2}$ optical constants were taken from figure $1 ; \mathrm{Nb}_{2} \mathrm{O}_{5}$, $\mathrm{Cr}_{2} \mathrm{O}_{3}, \mathrm{HfO}_{2}, \mathrm{WO}_{3}$, and $\mathrm{ZrO}_{2}$ optical constants were taken from reference 7; $\mathrm{Ta}_{2} \mathrm{O}_{5}$ data was taken from reference 8 , and $\mathrm{SrTiO}_{3}$ data is unpublished. 


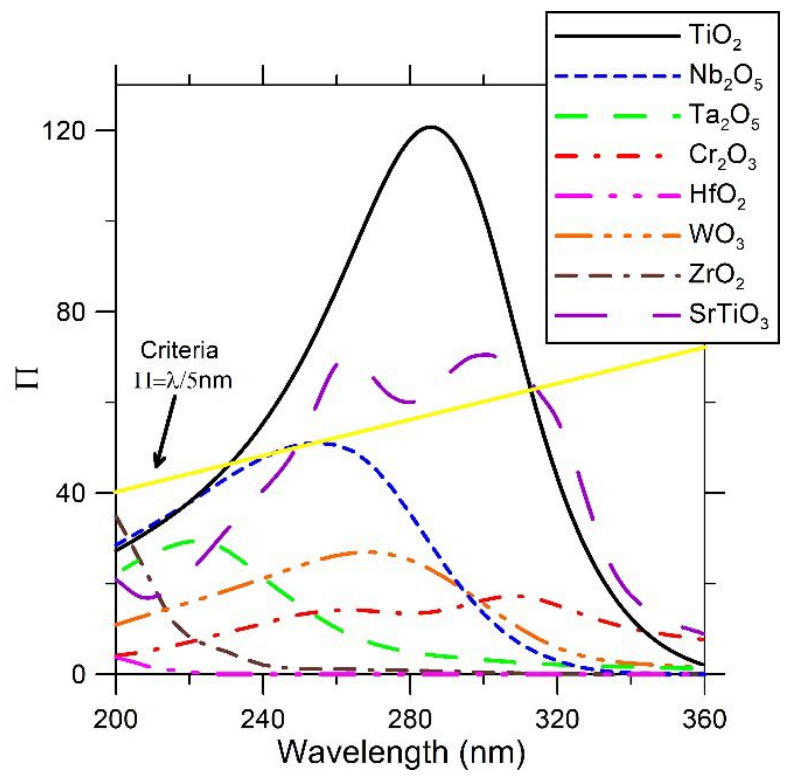

Fig. 2. Values of $\Pi=\left|\varepsilon_{G}\right| \cdot \operatorname{Im}\left(\varepsilon_{G}\right)$ for several oxides in the DUV spectral range

Figure 2 indicates that only $\mathrm{TiO}_{2}$ and $\mathrm{SrTiO}_{3}$ satisfy the condition $\Pi \geq \frac{\lambda}{5 \mathrm{~nm}}$ in the DUV. $\mathrm{TiO}_{2}$ presents the highest values of $\Pi$ function in the $\sim 220-320 \mathrm{~nm}$ range, which is coincident with the location of its absorption peak caused by interband transitions. Longwards of $\sim 320 \mathrm{~nm}$, although $\mathrm{TiO}_{2}$ presents high $|\varepsilon|$ due to the high values of $\varepsilon_{1}$, the absorption (as it becomes transparent above $\sim 340 \mathrm{~nm}$ ) turns it unsuitable for the development of efficient WGP. Similar effect can be seen shortwards of $230 \mathrm{~nm}$, where even though absorption of $\mathrm{TiO}_{2}$ is still high, $\varepsilon_{1}$ becomes small, and so does $\Pi$.

\subsection{Design using an Effective Medium Theory}

WGP was designed using the effective medium theory (EMT) proposed by Rytov ${ }^{9}$ for complex dielectric function of periodic structures. This EMT is valid if the period of the grating is shorter than the incident wavelength; in that case, the periodic structure can be replaced by an anisotropic layer, whose TE and TM optical constants are given by the following secondorder EMT expressions ${ }^{10}$ :

$$
\begin{aligned}
& \varepsilon^{(2)}{ }_{e f f, T E}=\varepsilon^{(0)}{ }_{e f f, T E}+\frac{\pi^{2}}{3} f^{2}(1-f)^{2}\left(\varepsilon_{G}-\varepsilon_{V}\right)\left(\frac{\mathrm{P}}{\lambda}\right)^{2} \\
& \varepsilon^{(2)}{ }_{\text {eff }, T M}=\varepsilon^{(0)}{ }_{\text {eff }, T M}+\frac{\pi^{2}}{3} f^{2}(1-f)^{2}\left(\frac{1}{\varepsilon_{G}}-\frac{1}{\varepsilon_{V}}\right)^{2}\left(\varepsilon^{(0)}{ }_{\text {eff }, T M}\right)^{3}{ }^{(0)}{ }_{\text {eff }, T E}\left(\frac{\mathrm{P}}{\lambda}\right)^{2} \\
& \varepsilon^{(0)}{ }_{\text {eff,TE }}=f \varepsilon_{G}+(1-f) \varepsilon_{V} \\
& \varepsilon^{(0)}{ }_{\text {eff,TM }}=\frac{\varepsilon_{G} \varepsilon_{V}}{f \varepsilon_{V}+(1-f) \varepsilon_{G}} \\
& \varepsilon_{e f f, T M}=\varepsilon^{(0)}{ }_{e f f, T M}+\varepsilon^{(2)}{ }_{e f f, T M} \text { and } \varepsilon_{e f f, T E}=\varepsilon^{(0)}{ }_{e f f, T E}+\varepsilon^{(2)}{ }_{e f f, T E}
\end{aligned}
$$


where $\lambda$ is the central wavelength of the target spectral range, $f$ is the ratio $w / P, w$ is the ridges width and $\mathrm{P}$ is the grating period, i.e. the sum between $w$ and the space between ridges. $\varepsilon_{G}$ and $\varepsilon_{V}$ are the complex dielectric constants of the grating material and of the voids, respectively; we assume that there is air or vacuum between the ridges in the grating, thus $\varepsilon_{V}=1+0 \mathrm{i}$. Superscripts (0) and (2) refers to zero-order and second-order of EMT, respectively.

WGP was designed using equations 1 and following the steps suggested by Liao and Zhao ${ }^{11}$ :

1) WGP works as zero-order grating. Thus, P must satisfy the following condition ${ }^{12}$ :

$$
\mathrm{P} \leq \frac{\lambda}{\sqrt{\varepsilon_{s}}}
$$

Where $\varepsilon_{\mathrm{s}}=1.58+0 \mathrm{i}$ is the average value of the dielectric constant of the fused silica substrate in the DUV range. From eq. (2) we obtain a maximum value of $P$, which has to be compatible with the practical fabrication limits.

2) WGP must work as an antireflection coating for TM polarization; thus, the AR dielectric constant condition is satisfied if:

$$
\varepsilon^{(0)}{ }_{\text {eff }, T M} \cong \sqrt{\varepsilon_{S}}
$$

If eqs. (2) and (1) are combined, we obtain a preliminary value of $f$. This value, along with the value of $\mathrm{P}$ obtained above give us $w$.

3) Height of ridges should meet the AR coating height criteria ${ }^{12}$, given by:

$$
z=\frac{1}{4 \sqrt{\varepsilon^{(0)}{ }_{e f f, T M}}} \lambda
$$

From eq. (3) we obtain a preliminary value of $z$.

4) The design has been so far optimized for TM polarization. Now, with the parameters obtained before ( $P$ and $f$ ), we calculate the throughput for TE polarization as a function of $z$. The final value of $z$ should be tailored between the optimum value of $z$ for TE polarization (related with the throughput) and the optimum value of $\mathrm{z}$ for TM polarization (related with the extinction ratio). 


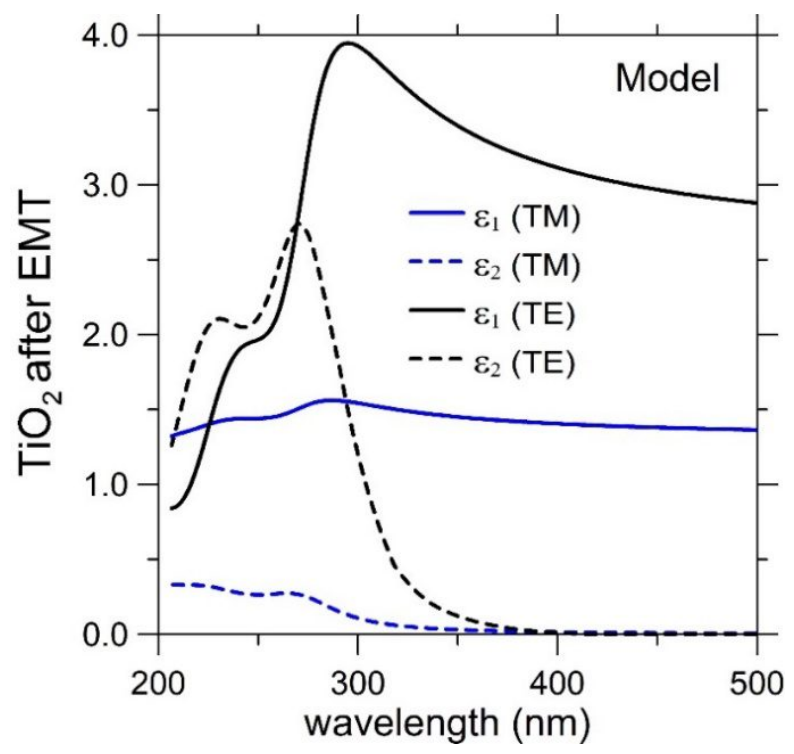

Fig. 3. Calculated values of $\varepsilon_{e f f, T M}$ and $\varepsilon_{e f f, T E}$ using EMT with $\mathrm{TiO}_{2}$ optical constants obtained in subsection 3.1, and $P=140 \mathrm{~nm}, \mathrm{f}=40 / 140$, and $\mathrm{z}=100 \mathrm{~nm}$. Calculations were done with IMD $^{13}$ software

Using the EMT, we have obtained a preliminary design (not shown), which is further optimized in the next subsection. Figure 3 shows $\varepsilon_{\text {eff,TM }}$ and $\varepsilon_{\text {eff,TE }}$ given by the final parameters $\mathrm{P}=140 \mathrm{~nm}, \mathrm{f}=40 / 140$, and $\mathrm{z}=100 \mathrm{~nm}$ determined in subsection 3.3. We can see through $\varepsilon_{\text {eff,TM }}$ that the grating acts as a fair AR coating for the substrate and with small absorption, meeting the condition given by eq. 3 for TM wave across the DUV range. On the other hand, the imaginary part of $\varepsilon_{\text {eff,TE }}$ presents large values across the range of interest, which is suitable for the absorption of TE radiation. EMT requires of short computation time and it is straightforward to optimize, but it might present inaccuracies as it is based on approximations.

\subsection{Design using Finite-Difference Time-Domain analysis}

Here we refine the preliminary design obtained in the previous subsection using EMT through FDTD analysis, which is more accurate but greatly time-consuming. A model for the WGP was created with OptiFDTD software ${ }^{14}$ using the set of parameters obtained with EMT as a starting point. OptiFDTD is a very powerful software that solves numerically the Maxwell's equations in both time and spatial domains, and gives the electric and magnetic field distributions at each time step in each point of the mesh where the device is modelled. This software is suitable for the simulation of the polarization effects of gratings.

Figure 4 presents the WGP layout built in this software. To obtain a domain-reduced simulation for periodic structures, a basic cell conforming a single period along the $\mathrm{X}$-axis for the grating was designed, consisting in a portion of the substrate and a single ridge. Boundaries of the basic cell were selected as Periodic Boundary Condition (PBC) along $X$ and $Y$ directions, acting as a perfect mirror, whereas the boundaries along $Z$ direction were selected 
as Anisotropic Perfectly Matched Layer (APML), where any wave crossing the latter is purely transmitted.

A Gaussian Modulated Continuous Wave ${ }^{15}$ (GMCW) was used as an excitation source. It was centered at $1100 \mathrm{THz}(\sim 273 \mathrm{~nm})$ in the frequency domain, and it is described by a half width of $5.25 \cdot 10^{-16} \mathrm{~s}$ and a time offset of $1.85 \cdot 10^{-15} \mathrm{~s}$. The polarization degree of the incident wave is zero. The direction of propagation of the GMCW is perpendicular to substrate normal. An observation line was placed inside the substrate, parallel to $X$ axis, to record the components of the electric and magnetic fields to compute the transmittance. As the substrate is absorption-free, the distance at which the observation line is placed is irrelevant for the result. In order to take into account the back face of the substrate in the final result, which is not included in the layout, intensities of transmitted waves were multiplied by the factor $\left(\frac{2 N s}{N s+1}\right)^{2} \frac{1}{N s}$ where Ns is the refractive index of the substrate. Figure 4 shows the configuration for the basic cell of WGP, the boundaries, the incident wave, and the observation line.
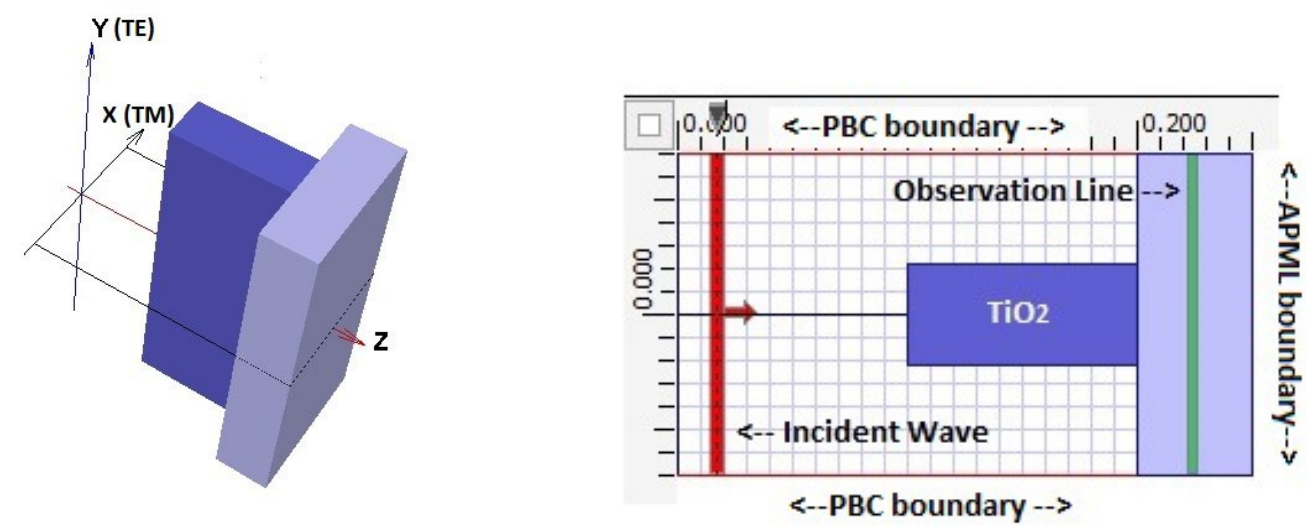

Fig. 4. 3D (left) and 2D (Right) cell unit model layout for the WGP

The basic cell was meshed with a step of $0.4 \mathrm{~nm}$ in all directions. The simulation was run in the time domain for 10000 time steps with a time step size of $8.9 \cdot 10^{-19} \mathrm{~s}$; this value for time step was selected to satisfy the Courant condition ${ }^{16}$ for a length step of $0.4 \mathrm{~nm}$. Once the simulation was completed in the time domain, the discrete Fourier transform was calculated to obtain the transmittance response as a function of frequency (or wavelength) for each polarization.

Following this procedure, the parameters $\mathrm{w}, \mathrm{P}$, and $\mathrm{z}$ obtained in subsection 3.2 were again optimized with the FDTD software, and the final design is $w=40 \mathrm{~nm}, P=140 \mathrm{~nm}$, and $z=100 \mathrm{~nm}$. Figure 5 shows the comparison between two designs using the aforementioned parameters, using EMT and FDTD for both TE and TM polarizations. Both designs present a good TM-to-TE contrast, with an average throughput of $>50 \%$ across the DUV range. The agreement between them is good, although some discrepancies can be seen in the short wavelengths where the period of the grating is comparable with the incident wavelength, so that the design using EMT is expected to present some inaccuracies. 
Additionally, far field calculations were done to calculate the dispersion loses at high angles from normal incidence, and we found that the relative amount of photons dispersed at angles above $10^{\circ}$ from normal incidence is $<10^{-5} \%$ at $\lambda=200 \mathrm{~nm}$, and smaller at longer wavelengths. Regarding the acceptance angle, far field calculations also shown that no diffraction orders (other than zero-order) are expected for incidence angles as high as $30^{\circ}$.
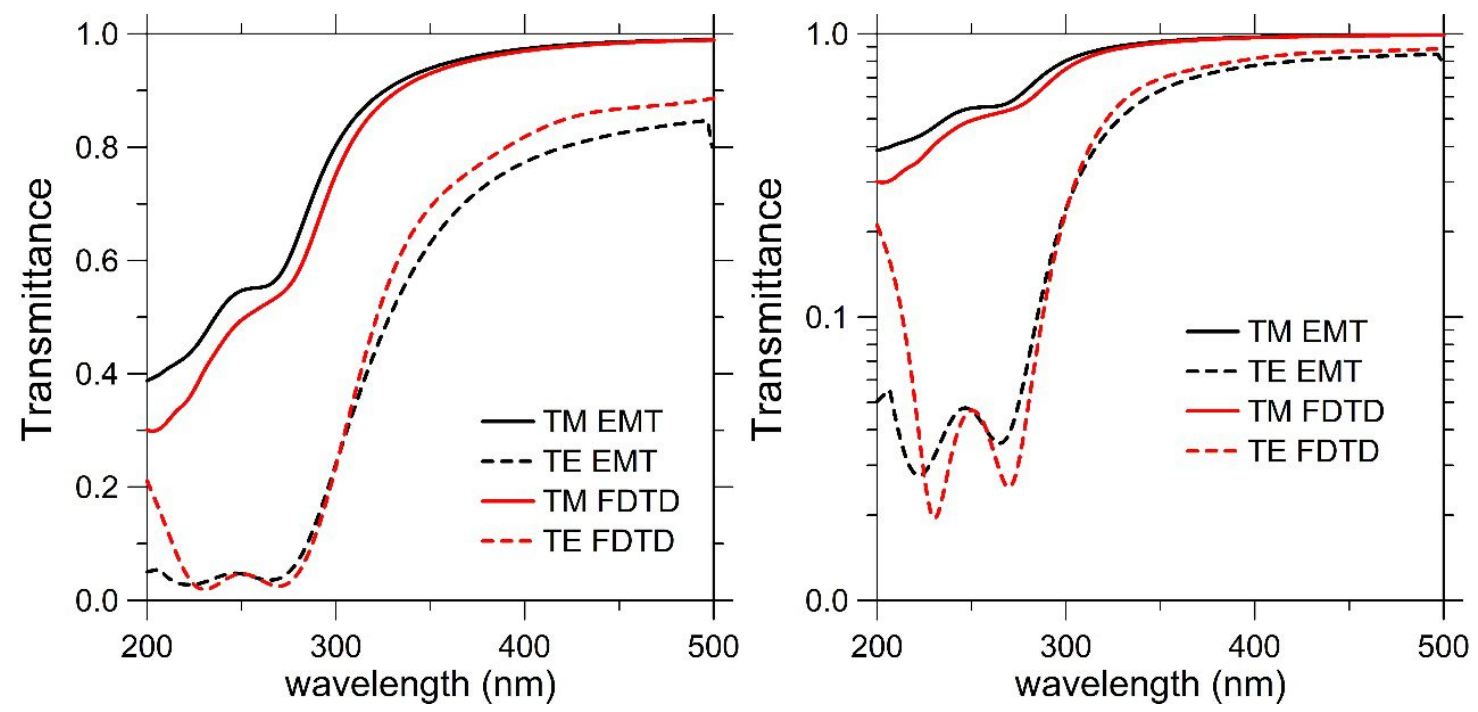

Fig. 5. Comparison between the design of a $\mathrm{TiO}_{2}$-based WGP using EMT and FDTD analysis. Parameters in both designs are $P=140 \mathrm{~nm}, \mathrm{w}=40 \mathrm{~nm}$, and height $=100 \mathrm{~nm}$. Left, lineal scale; right, logarithm scale

\section{FABRICATION}

The WGP prototype was made by electron beam followed by lift-off process. A thin positive photoresist film is deposited on a substrate, an inverse pattern is created by electrons exposure, and the photoresist is subsequently etched. Then, the target material is deposited on top of the photoresist, so that it can reach the surface of the substrate only in the regions where the photoresist was previously etched. Finally, the photoresist is dissolved and the target material on top is lifted-off, whereas the target material remains only in the regions where it has a direct contact with the substrate. A sketch of the lift-off procedure is shown in figure 6. 


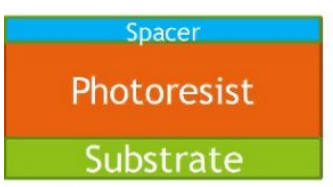

Photoresist (PR) and SPACER spin-coated

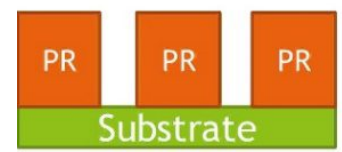

PR development

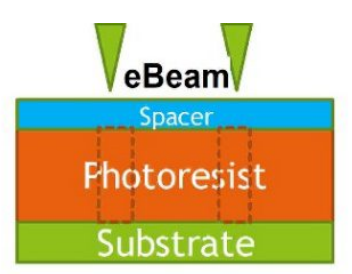

PR exposure

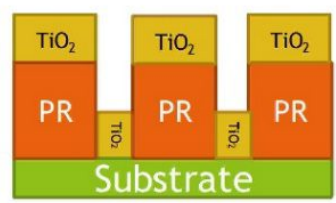

$\mathrm{TiO}_{2}$ deposition

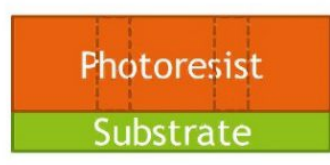

SPACER removal

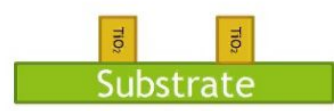

PR lift-off

Fig. 6. Sketch of lift-off process main steps. All objects in this sketch are not to scale.

The photoresist patterning process was carried out at Data Storage Institute (DSI-A*STAR Singapore). A positive ZEP-520A photoresist was spin-coated on a fused silica substrate (10 $\mathrm{mm} \times 10 \mathrm{~mm}$ size, $1 \mathrm{~mm}$ thickness) at $5000 \mathrm{rpm}$ to obtain a $300 \mathrm{~nm}$ thick layer and baked at $180{ }^{\circ} \mathrm{C}$ for $2 \mathrm{~min}$; photoresist must be significantly thicker than the thickness of $\mathrm{TiO}_{2}$ being lifted-off. Then, sample was spin-coated again with ESPACER at $1000 \mathrm{rpm}$, and baked at $100^{\circ} \mathrm{C}$ for $1 \mathrm{~min}$; ESPACER coating was used to reduce charging effect in sample. Then, four different fields of $1 \mathrm{~mm} \times 1 \mathrm{~mm}$ area on sample were exposed to electron beam working with a $100 \mathrm{pA}$ current at different dosages of $0.1 \mu \mathrm{s} / \mathrm{dot}, 0.15 \mu \mathrm{s} / \mathrm{dot}, 0.2 \mu \mathrm{s} / \mathrm{dot}$, and $0.25 \mu \mathrm{s} / \mathrm{dot}$. After the exposure, ESPACER layer was removed using deionized (DI) water, and the exposed areas of the positive resist were developed using 0 -xylene bath for 60s.

The sample with the inverse pattern was brought back to SSLS and loaded in PLD chamber, where a $\mathrm{TiO}_{2}$ layer of $\sim 100 \mathrm{~nm}$ was deposited on the patterned photoresist at room temperature in a $10 \mathrm{mTorr} \mathrm{O}_{2}$ pressure. After deposition, sample was extracted from PLD and, at SSLS microfabrication facility, the photoresist was lifted-off with three acetone rinses of 5 minutes each in an ultrasonic bath at room temperature. Next, sample was rinsed 5 minutes two times with isopropyl alcohol, then rinsed 5 minutes once with DI water, and finally sample was blown with dry nitrogen. Once the lift-off process was completed, the sample was annealed in vacuum at $500^{\circ} \mathrm{C}$ in a $10 \mathrm{mTorr}_{2}$ pressure.

The four patterned fields were inspected before and after $\mathrm{TiO}_{2}$ deposition and lift-off with a Leica DMLM optical microscope using a magnification of 500x (bright field). Before lift-off, the interferential color of fields patterned with a dosage of $0.1 \mu \mathrm{s} / \mathrm{dot}$ and $0.15 \mu \mathrm{s} /$ dot seemed relatively uniform, whereas fields patterned with a dosage of $0.2 \mu \mathrm{s} / \mathrm{dot}$ and $0.25 \mu \mathrm{s} / \mathrm{dot}$ presented non-uniformities in color and several wide stray lines partially resolved by microscopy. After lift-off, fields patterned with $0.1 \mu \mathrm{s} /$ dot and $0.15 \mu \mathrm{s} /$ dot completely disappeared, which might be caused by an incomplete photoresist development due to underexposure. Fields patterned with $0.20 \mu \mathrm{s} /$ dot and $0.25 \mu \mathrm{s} /$ dot presented blank areas (bare 
substrate with no $\mathrm{TiO}_{2}$ lines on it) which attained a total relative surface in the $1 \mathrm{~mm} \times 1 \mathrm{~mm}$ patterned field of $35 \%$ and $5 \%$, respectively. Besides, a relative surface of $\sim 1 \%$ was covered with stray lines (mostly oriented following TE direction) in the field patterned with $0.20 \mu \mathrm{s} / \mathrm{dot}$, whereas it was $\sim 9 \%$ in the $0.25 \mu \mathrm{s} /$ dot field (mostly following erratic directions). These results are summarized in table 1 . Samples were also characterized by AFM. AFM inspection showed that the density of ridges became smaller in the proximity of blank areas, particularly in the $0.20 \mu \mathrm{s} / \mathrm{dot}$ field. This adverse effect, along with the presence of wide stray lines and blank areas might have been induced by the charging effect of substrate, producing a deflection of the electron beam and hence an irregular exposure.

Table 1. Parameters of the four patterned fields after lift-off process. Microscope images were analyzed with Image ${ }^{17}$ software

\begin{tabular}{|c|c|c|c|}
\hline Dosage ( $\mu \mathrm{s} / \mathrm{dot})$ & $\begin{array}{c}\text { Field size } \\
\left(\mathrm{mm}^{2}\right)\end{array}$ & $\begin{array}{c}\text { \% of field blank } \\
\text { after lift-off }\end{array}$ & $\begin{array}{c}\text { \% of surface covered by stray } \\
\text { lines ( resolved by microscopy) }\end{array}$ \\
\hline 0.1 & 1 & $100 \%$ & - \\
\hline 0.15 & 1 & $100 \%$ & - \\
\hline 0.20 & 1 & $35 \%$ & $1 \%$ (mostly oriented) \\
\hline 0.25 & 1 & $5 \%$ & $9 \%$ (erratic directions) \\
\hline
\end{tabular}

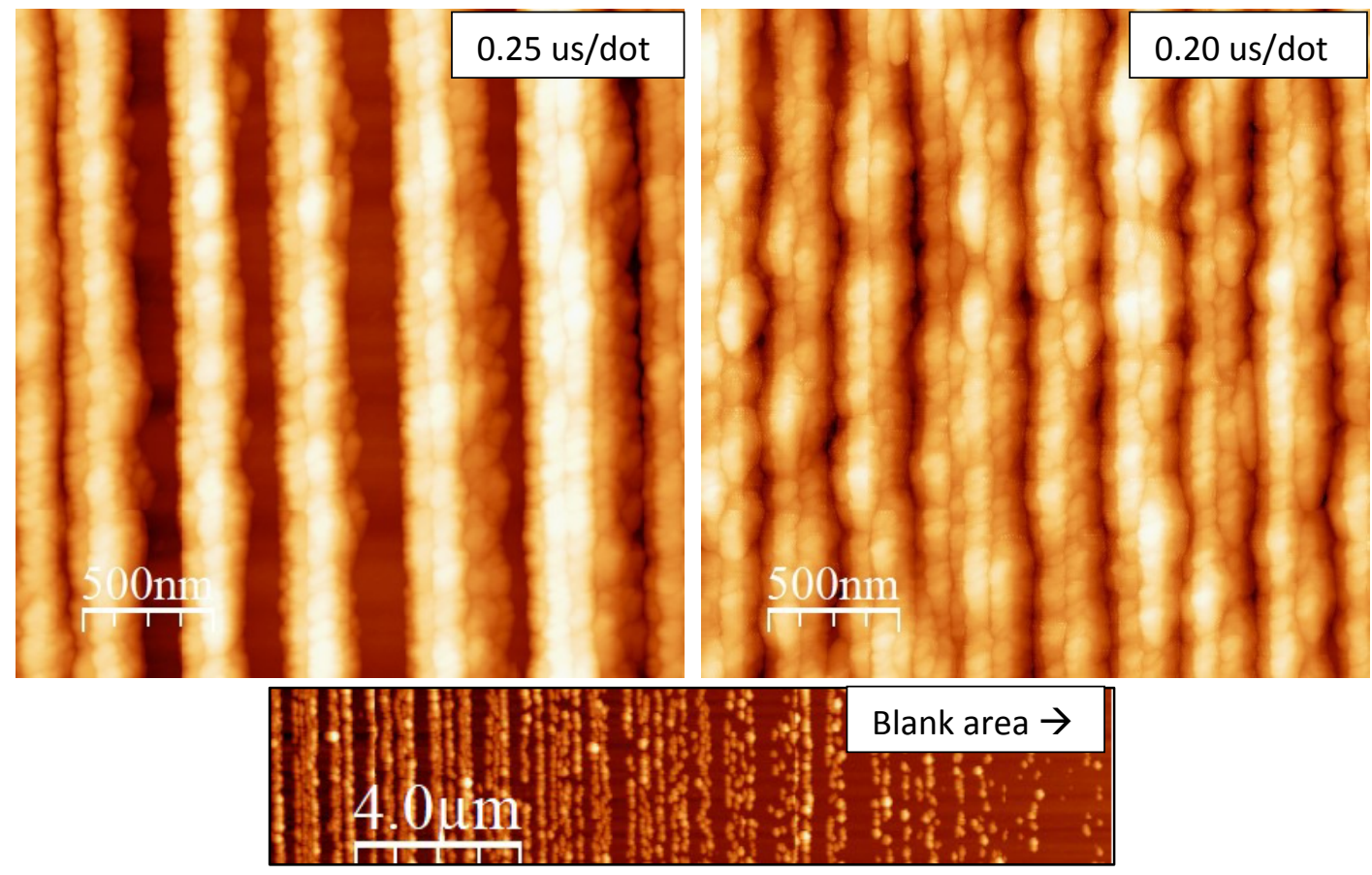



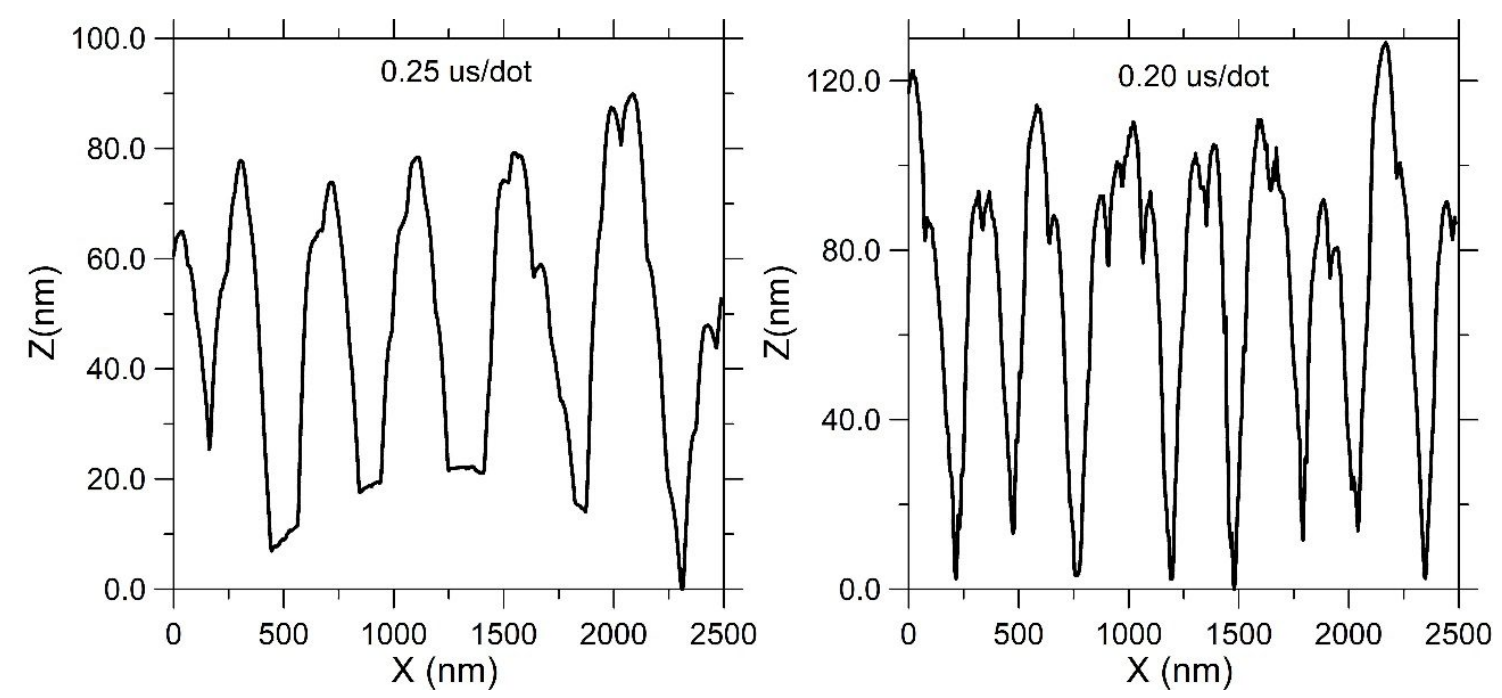

Fig. 7. AFM images (top) and cross-section profiles (bottom) of selected areas within the fields patterned with a dosage of $0.25 \mu \mathrm{s} / \mathrm{dot}$ (left) and $0.20 \mu \mathrm{s} /$ dot (right). The small image in the middle shows how the density of $\mathrm{TiO}_{2}$ lines decreases and the space between lines increases, as we move towards the blank areas within the patterned field areas. Profiles were obtained with WSxM software ${ }^{18}$

Figure 7 displays the AFM pictures (top) and surface profiles (bottom) of selected areas, taken away from blank areas, in the fields patterned with a dosage of $0.25 \mu \mathrm{s} / \mathrm{dot}$ (left) and 0.20 $\mu \mathrm{s} /$ dot (right). Averaged experimental P and w parameters were determined from the power spectral density function, which was calculated from AFM topography images. Even though $\mathrm{TiO}_{2}$ ridges in both fields present substructures with a period of $\mathrm{P}=\sim 140 \mathrm{~nm}$, which is the designed value, the experimental profile is noticeably different from the design. The dominant period values found are $P=\sim 430 \mathrm{~nm}, \mathrm{~W}=\sim 230 \mathrm{~nm}(25 \mu \mathrm{s} /$ dot field), and $\mathrm{P}=\sim 320 \mathrm{~nm}, \mathrm{~W}=\sim 200$ $\mathrm{nm}(0.20 \mu \mathrm{s} /$ dot field). These large dominant structures have periods larger than DUV wavelengths, so that the sub-wavelength condition is lost and photons will be undesirably lost in higher diffraction orders in the transmission measurements. We foresee a poor polarization performance according to FDTD simulations using the experimental profiles shown in figure 7 . There is plenty of room for improvement in our fabrication process.

\section{CHARACTERIZATION}

Samples were preliminary characterized by spectroscopic ellipsometry to obtain the effective dielectric constant for both TE and TM orientations. Ellipsometry measurements at $50^{\circ}$ from normal incidence were carried out on fields patterned with $0.25 \mu \mathrm{s} / \mathrm{dot}$ and 0.20 $\mu \mathrm{s} /$ dot. Experimental data was fitted with Tauc-Lorentz model along with two additional Lorentz oscillators, and the effective TE and TM dielectric constants derived from the fitting are shown in figure 8 . Measurements shortwards of $215 \mathrm{~nm}$ were noisy due to the use of the focusing lenses, hence data was only fitted in the $215-500 \mathrm{~nm}$ range. 

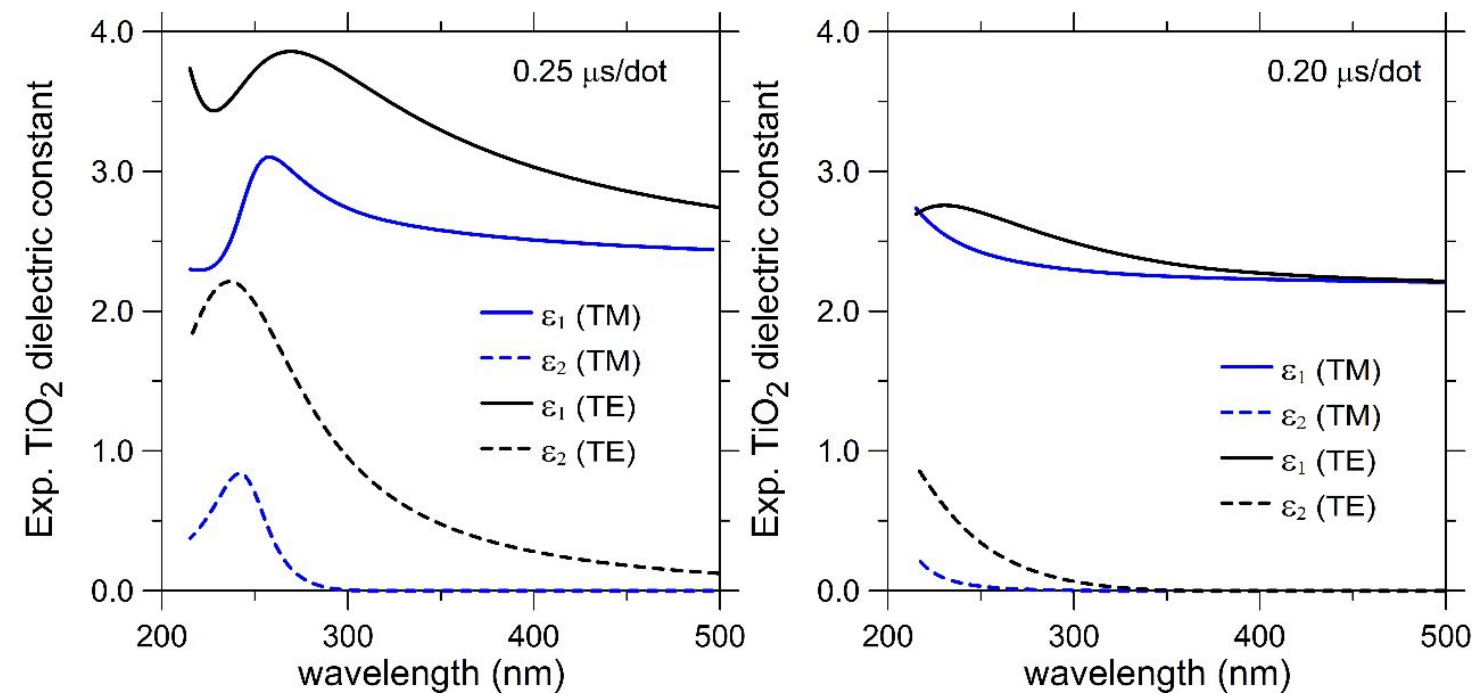

Fig. 8. Experimental TE and TM values of the effective dielectric constant obtained in the fields patterned with a dosage of $0.25 \mu \mathrm{s} /$ dot (left) and $0.20 \mu \mathrm{s} /$ dot (right)

If we compare the experimental values shown in figure 8 with the theoretical values of figure 3, we find that the TE and TM dielectric constant contrast is smaller than predicted, particularly in the field patterned with $0.20 \mu \mathrm{s} / \mathrm{dot}$, where both TE and TM components of the complex dielectric constant resembles to fused silica with extra absorption. This poor contrast is due to the large blank surface without $\mathrm{TiO}_{2}$ ridges present in the aforementioned field. Contrarily, contrast is much better in the field patterned with $0.25 \mu \mathrm{s} / \mathrm{dot}$, particularly at short wavelengths. The effective TE dielectric constant in the latter field is compatible with the design, whereas TM component is larger than in the design, hence limiting his AR property. TE and TM components of the normal incidence transmittance are yet to be measured.

\section{CONCLUSIONS AND CURRENT WORK}

In conclusion, we have introduced (and used) a new merit function $\Pi$ to select the optimum material for WGPs in the DUV range; among the oxides analysed, $\mathrm{TiO}_{2}$ was the best. Then, we have presented designs following two different paths: using an EMT and with FDTD analysis. Both designs shown an average transmittance above $50 \%$ in DUV range along with good TE-to-TM contrast, and an good agreement between them. We show negative results on our first prototype of WGP for the DUV range. It was fabricated by e-beam lithography followed by lift-off process, using four different dosages to pattern the photoresist. We found that the two fields patterned with the lower dosages were underexposed, leaving a blank surface after lift-off process, and the two fields with the higher dosages were not uniform due to substrate charge. AFM pictures of the latter fields showed a morphology quite different from the design, presenting substructures with a period of $\sim 140 \mathrm{~nm}$ but also dominant structures with larger periods, along with blank areas and stray lines. TE and TM effective dielectric constant of the $\mathrm{TiO}_{2}$ fields patterned with dosages of $0.20 \mu \mathrm{s} / \mathrm{dot}$ and $0.25 \mu \mathrm{s} / \mathrm{dot}$ were obtained by spectroscopic ellipsometry. The experimental values of the dielectric 
constant contrast is poor in the field patterned with $0.20 \mu \mathrm{s} /$ dot, however it is much better in the one patterned with $0.25 \mu \mathrm{s} /$ dot dosage, even though the contrast is smaller than in the design.

Nowadays, we plan to measure TE and TM transmittances of the device using the transmission mode configuration in our Woollam V-VASE ellipsometer. On the fabrication side, we are working in the optimization of the e-beam exposure of the positive photoresist in order to minimize the substrate charging effect; we also are exploring an alternative patterning procedure, e-beam lithography followed by RIE process.

\section{ACKNOWLEDGMENTS}

We thanks Toh Yeow Teck from Data Storage Institute (A*STAR) for sample patterning. We acknowledge Singapore Synchrotron Light Source for providing the facility necessary for conducting the research. The Laboratory is a National Research Infrastructure under the National Research Foundation, Singapore.

\footnotetext{
${ }^{1}$ Z. Yong, P. E. Trevisanutto, L. Chiodo, I. Santoso, A. R. Barman, T. C. Asmara, S. Dhar, A. Kotlov, A. Terentjevs, F. Della Sala, V. Olevano, M. Rübhausen, T. Venkatesan, and A. Rusydi, "Emerging giant resonant exciton induced by Ta substitution in anatase TiO2: A tunable correlation effect," Phys. Rev. B, 93, 205118 (2016)

${ }^{2}$ K. Asano, S. Yokoyama, A. Kemmochi, and T. Yatagai, "Fabrication and characterization of a deep ultraviolet Wire Grid Polarizer with a chromium-oxide subwavelength grating," Appl. Opt. 53, 13, 2942 (2014)

${ }^{3}$ E. Gardner, B. Wang, M. Davis, and J. D. Dredge Jr, "Durable, inorganic, absorptive ultra-violet grid polarizer," US Patent US8947772B2 (2015)

${ }^{4}$ T. Siefke, S. Kroker, K. Pfeiffer, O. Puffky, K. Dietrich, D. Franta, I. Ohlidal, A. Szeghalmi, E-B. Kley, and A. Tunnermann, "Materials pushing the applications limits of Wire Grid Polarizers further into the deep ultraviolet spectral range," Adv. Optical Mater. 4, 1780 (2016)

${ }^{5}$ H. R. Philipp, "Optical properties of non-crystalline Si, SiO, SiOx and SiO2," J. Phys. Chem. Solids 32, 1935-1945 (1971).

${ }^{6}$ G. E. Jellison and F. A. Modine, "Parameterization of the optical functions of amorphous materials in the interband region," Appl. Phys. Lett. 69(3), 371-373 (1996)

${ }^{7}$ Nanolithography Research Labs, Rochester Institute of Technology, optical constants database at www.rit.edu/kgcoe/microsystems/lithography/thinfilms/thinfilms/thinfilms.html

${ }^{8}$ L. V. Rodríguez-de Marcos, J. I. Larruquert, J. A. Méndez, and J. A. Aznárez, "Self-consistent optical constants of $\mathrm{SiO}_{2}$ and $\mathrm{Ta}_{2} \mathrm{O}_{5}$ films," Opt. Mater. Express 6, 3622-3637 (2016)

${ }^{9}$ S. M. Rytov, "Electromagnetic Properties of a Finely Stratified Medium," J. Exper. Sov. Phys. 29, 605 (1955)

${ }^{10} \mathrm{~S}$. Moon and D. Kim, "Fitting-based determination of an effective medium of a metallic periodic structure and application to photonic crystals," JOSA A 23, 199 (2006)

${ }^{11}$ Y-L. Liao and Y. Zhao, "Design of wire-grid polarizer with effective medium theory," Opt. Quant. Electron. 46, 641 (2014)

${ }^{12}$ M. Born and E. Wolf, "Principles of Optics: Electromagnetic Theory of Propagation, Interference and Diffraction of Light," Cambridge: Cambridge University Press (1999)

${ }^{13}$ D. L. Windt, "IMD: Software for modeling the optical properties of multilayer films," Computers in Physics 12, 360-370 (1998)

${ }^{14}$ OptiFDTD, https://optiwave.com

${ }^{15}$ OptiFDTD_32-bit_Technical_Background, available at https://optiwave.com
} 
${ }^{16}$ R. Courant, K. Friedrichs, H. Lewy, "Über die partiellen Differenzengleichungen der mathematischen Physik," Mathematische Annalen, 100 (1), 32-74 (1928)

${ }^{17}$ Imagej, https://imagej.nih.gov/ij/docs/index.html

${ }^{18}$ I. Horcas, R. Fernanced, J. M. Gomez-Rodriguez, J. Colchero, J. Gomez-Herrero, and A. M. Baro, "WSXM: A software for scanning probe microscopy and a tool for nanotechnology," Review of Scientific Instruments 78, 013705 (2007) 